\title{
LA NATURE DU SOL ET SON INFLUENCE SUR L'ÉCONOMIE ET LE PEUPLEMENT D’UNE RÉGION GENEVOISE, LA CHAMPAGNE
}

\section{Georges Lobsiger}

L'étude des modifications de la répartition de la population genevoise de 1900 à 1957 a permis de déceler deux zones principales de communes en voie de dépeuplement: le Nord-Est, avec les communes d'Hermance, Anières, Corsier et Gy, et le Sud-Ouest avec les communes de Chancy, Avully, Cartigny, Aire-la-Ville, Avusy, Laconnex et Soral. Deux communes isolées se dépeuplent également (Collex-Bossy et Céligny) alors que les communes de Dardagny et Choulex n'ont pas évolué démographiquement. Seule la région du Sud-Ouest sera examinée ici.

Son nom, la Champagne est extrapolé par la coutume, alors que sous l'ancien régime, soit avant 1814, seules les communes de Chancy, Cartigny et Avully répondaient à ce nom. Le Traité de Turin du 16 mars 1816 accorda au nouveau canton suisse des territoires contestés depuis des siècles, terres qui avec celles acquises de la France par le Traité de Paris d'octobre 1814 accordèrent à la République et Canton de Genève la frontière linéaire de type moderne qui avait toujours fait défaut à la Ville et République de Genève, dont les dépendances étaient enclavées dans les royaumes de France et de Sardaigne. Les communes de Airela Ville (avec 175 habitants), et Avusy (avec 1201 habitants) augmentèrent la surface genevoise de 1189 hectares. En 1847, Avusy se scindera et formera les communes de Soral et Avusy. En 1850, Soral se divisera en Soral et Laconnex. Ces divisions de communes furent fréquentes jusqu'en 1869 et caractérisent les communes ex-sardes rattachées à Genève. En 1816, le Canton de Genève comptait 48671 habitants, dont 25742 citadins et 22928 ruraux. Sa densité était alors de 200 habitants au kilomètre carré, alors qu'aujourd'hui chacun de ses $2+6 \mathrm{~km}^{2}$ porte 927 habitants.

La Champagne en 1816 comptait 2567 habitants fixés sur $29 \mathrm{~km}^{2}$ (chiffre arrondi), alors qu'aujourd'hui (fin 1957) elle ne compte plus que 1911 habitants, passant du $5,28 \%$ de la population cantonale à $0,8 \%$ de cette population. Que s'est-il passé? Nous ne sommes cependant qu'entre 7 et 15 kilomètres du centre de la ville. Ici la nature du sol a joué un rôle prépondérant, assisté par le phénomène mondial d'urbanisation et de fuite de la terre. Il ne peut être question ici de céder à quelque envoûtement déterministe et si le fait géographique peut expliquer des faits de régression dans des zones éloignées, ici, nous sommes presque dans la banlieue d'une capitale, qui voit sa population augmenter à un rythme inquiétant, surtout par immigration.

Il est intéressant d'examiner une zone que l'on pourrait emphatiquement nommer «sousdéveloppée naturellement» à une si courte distance de ses marchés. L'histoire du peuplement de Genève laisse supposer que les sols pauvres de ce terroir n'ont guère attiré les colons.

La surface du Canton, sans le lac, est de $2+6,+15+49 \mathrm{~km}^{2}$ et sa populations atteint 227590 habitants. Mais nous éliminerons de nos calculs ultérieurs la ville et les 4 grandes communes suburbaines (Vernier, Carouge, Chêne-Bougeries et Lancy) pour ne conserver que la surface et la population du canton que nous nommerons rural, malgré le processus très rapide d'urbanisation de communes à bonnes terres. Dorénavant, lorsque nous dirons «canton» il s'agira donc uniquement de ce canton sans la ville et l'agglomération. La surface de ce canton atteint $211,459879 \mathrm{~km}^{2}$ et sa population est de 37323 habitants, ce qui donne une densité de 176 habitants au kilomètre carré, Champagne comprise.

Nous avons vu la forte population de la Champagne en 1816; voyons maintenant la situation de 1900 et celle de 1957 , qui seule peut nous intéresser.

En 1900, la population de la Champagne s'élevait à 2404 habitants, donc une densité de $83 \mathrm{hab} / \mathrm{km}^{2}$, alors que 1957 présente 1911 habitants seulement pour ses $28,995333 \mathrm{~km}^{2}$ (arrondi $29 \mathrm{~km}^{2}$ ) ; donc une densité de $66 \mathrm{hab} / \mathrm{km}^{2}$ caractérise aujourd'hui ce petit pays. Ces $29 \mathrm{~km}^{2}$ représentent le $14,15 \%$ de la superficie du Canton et sa population le $5,1 \%$ de ce Canton (rural il va sans dire). La différence est notable. En 1900, la densité des communes rurales moyenne était de $108 \mathrm{hab} / \mathrm{km}^{2}$, aujourd'hui elle s'élève à 176 , comme dit plus haut, alors que la densité de la Champagne a passé de 83 à 66, la population ayant diminué en 57 ans jusqu'au 75,33\% de sa population de 1900. Mais si l'ont tient compte du cœfficient d'augmentation de 
la population rurale du Canton, on devrait obtenir pour la Champagne un chiffre théorique de 3750 habitants au lieu des 1911 actuels, ce qui fait une perte théorique de $50 \%$. La cause du dépeuplement de cette agréable région genevoise peut être expliquée en partie par la nature du sol, dont les déficiences se sont répercutées sur l'économie, ici presqu'exclusivement agricole. Ces difficultés n'apparaissent que dans le calcul et les aimables villages bien soignés, les cultures minutieuses, ne trahissent pas au promeneur les problèmes de production et les déficits créés par un sol ingrat.

La Champagne se compose d'un vaste plateau, réuni aux terrasses du Rhône par des pentes herbeuses et vives. En plusieurs points, tels Cartigny et Epeisses, des falaises de $85 \mathrm{~m}$. bordent ce plateau sur lequel sont bâtis de vieux villages, La PetiteGrave, Cartigny, Epeisses, Avully, Avusy, Athenaz, Sezegnin, Soral, ou des hameaux comme Passeiry et Champlong, tous à la limite du plateau à graviers et des pentes argileuses; seul Chancy est établi au milieu d'une ancienne terrasse du Rhône.

Ce plateau se'compose de graviers, restes d'un delta témoin d'un système hydrographique post-glaciaire aujourd'hui disparu. Les terrasses du Rhône sont naturellement graveleuses. L'argile glaciaire, le «diot» genevois, se trouve géographiquement entre les graviers des plateaux et ceux des terrasses alors que géologiquement il leur est inférieur. Citons encore quelques affleurements de molasse, de conglomérats et de sablons.

Les alluvions des plateaux couvrent $11,70 \mathrm{~km}^{2}$ soit le $40,48 \%$ de la surface
les alluvions des terrasses
soit une surface de

Les graviers apparaissent presqu'exclusivement sur les parties plates, donc sous agriculture, sur une surface d'environ $60 \%$ de la région envisagée. L'argile glaciaire $(33,70 \%)$ compacte, forme les pentes et elle est surtout sous herbages, naturels et artificiels. Quelques taillis sans grande valeur, recouvrent des zones argileuses, et les bancs de molasse et de conglomérats ne jouent aucun rôle agricole ni industriel. Les sablons très fertiles se trouvent dans la partie orientale de Soral, en dehors du complexe géologique et pédologique envisagé, mais il faut en tenir compte, puisque politiquement cette demi-commune de Soral riche en sablons fait partie de la Champagne.

On peut estimer à 3 mètres l'épaisseur des graviers sur les plateaux, reposant sur des sablons, de l'argile et des alluvions fluvio-glaciaires (Petite-Grave); on compte 3 à $4 \mathrm{~m}$. de graviers dans la région d'Avully, et à Avusy et Champlong, les sables ont jusqu'à $12 \mathrm{~m}$. d'épaisseur. A Chancy, $7 \mathrm{~m}$. de graviers reposent sur de l'argile. Les sondages effectués par Joukowsky dans la région de Soral pour atteindre la nappe d'eau souterraine ont traversé $2,30 \mathrm{~m}$. de limons et $2,60 \mathrm{~m}$. de graviers avant d'atteindre l'argile et des graviers alternés.

Nous sommes donc en présence d'un terrain essentiellement perméable, reposant sur des argiles compactes. L'infiltration des eaux de pluie est donc facilitée à un degré abusif par la nature du sol et l'on prétend, dans les milieux agricoles, qu'il faudrait une pluie tous les deux jours pour permettre aux céréales de se développer harmonieusement. Les années sèches sont néfastes à la Champagne. Cette ultra-porosité du sol doit retenir notre attention. Les villages ont été normalement construits à la limite des graviers et des pentes argileuses, donc près des affleurements possibles des nappes phréatiques. 


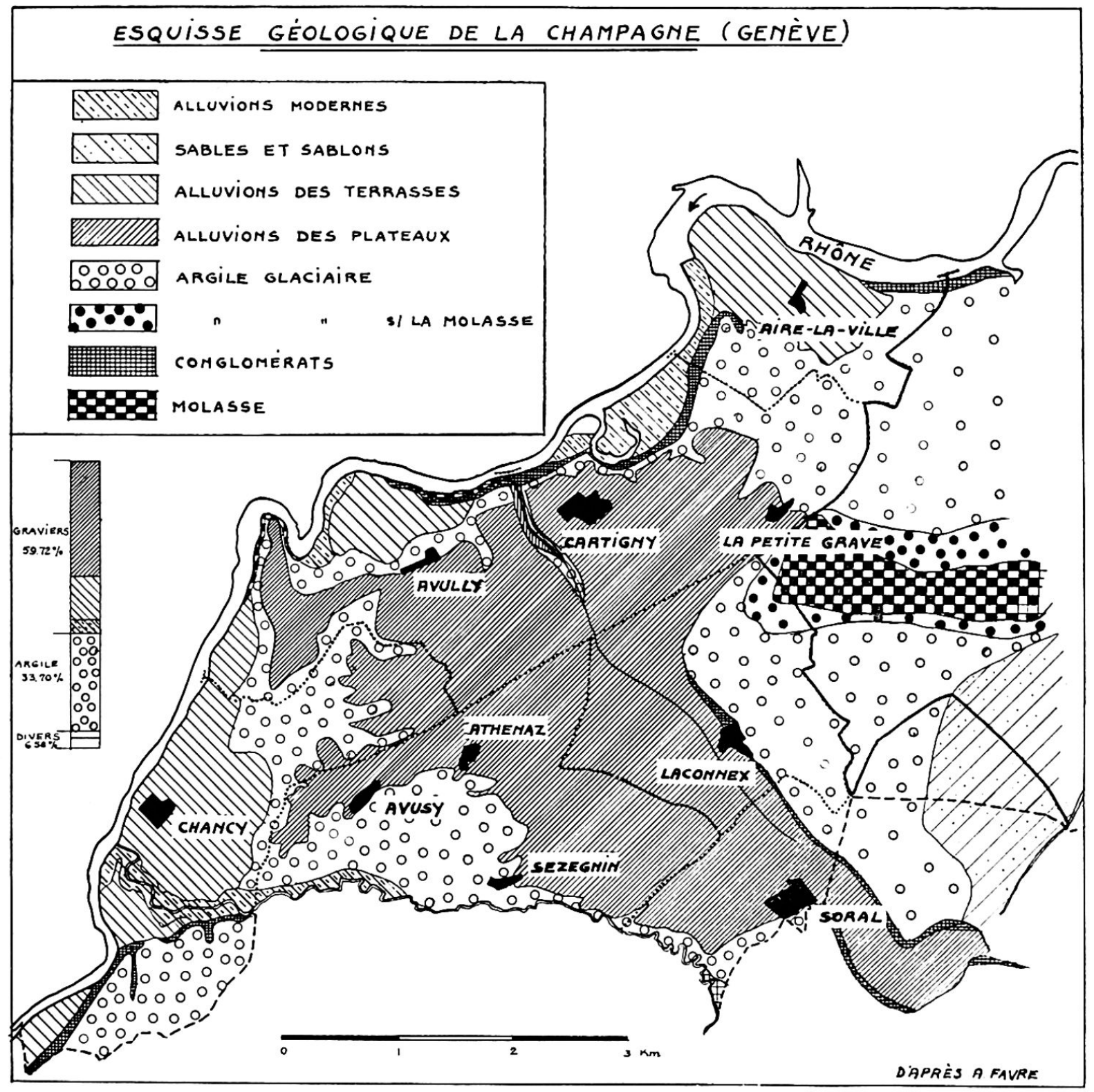

Les rendements agricoles souffrent de cet état de choses. Le Cercle des Agriculteurs de Genève, qui fonctionne officiellement en tant que Centrale cantonale pour la prise en charge des blés indigènes, estime, sous toutes réserves, que la moyenne de la Champagne en ce qui concerne le blé, est de 1700 kilos à l'hectare, alors que les terres fortes du canton fournissent en moyenne 2500 kilos, quelques communes atteignant 2800 kilos par hectare. Les pommes de terre obtiennent des rendements estimés moyennement de 15000 kilos par hectare alors que la moyenne cantonale atteint 20000 kilos.

Les agriculteurs de la Champagne, fortement enracinés au sol sont aussi bien équipés que leurs collègues des terres riches, mais ils ne sont pas autant rétribués qu'eux par hectare-travail. L'analyse de quelques éléments du récent recensement fédéral de entreprises, encore inédit, permet de faire quelques constatations intéressantes sur l'influence de la nature du sol sur la structure économique et démographique d'une région donnée.

Le canton (rural) exploite le $59,10 \%$ de sa surface et la Champagne utilise le $63,79 \%$ de son sol. Le canton compte 1403 exploitations dirigées par 1855 exploitants, avec au total 5684 personnes actives dans l'agriculture, ce qui donne une super- 
ficie de 9,962 hectares par exploitation. La Champagne, elle, compte 180 exploitations dirigées par 255 exploitants, avec une population active dans l'agriculture de 635 personnes. Chaque exploitation rurale y couvre $11, .477$ hectares, soit $116 \%$ d'une exploitation moyenne cantonale. Il faut dire que la monoculture ou la bi-culture de la Champagne ne connait pas les nombreuses petites exploitations maraîchères et horticoles de Genève, ce qui peut fausser légèrement le calcul des superficies comparées.

$15 \%$ de la population du canton rural travaille la terre, et les 59,10\% utilisés le sont pour $36,30 \%$ en agriculture et $22,80 \%$ en herbages, alors que la Champagne utilise $38,34 \%$ de son sol en agriculture et $25,45 \%$ en herbages (au total 63,79\%). On voit que la Champagne est très rurale puisque le $33 \%$ de sa population est active dans cette branche économique.

Si l'on veut poursuivre ces calculs inspirés par la nature du sol et son influence sur l'économie, on verra que chaque paysan genevois cultive par tête $2203 \mathrm{~m}^{2}$ (1350 $\mathrm{m}^{2}$ sous labours et $853 \mathrm{~m}^{2}$ sous herbe), alors que son collègue de la Champagne, cultive $2898 \mathrm{~m}^{2}$ (1763 $\mathrm{m}^{2}$ sous labours et $1135 \mathrm{~m}^{2}$ sous herbe) ce qui donne un rapport de $131 \%$ en sa faveur, rapport qui semble contrebalancer les déficits de production. La machinerie agricole permet au paysan de la Champagne de mener à bien cet excédent de travail. Le revenu-terre est donc plus faible en Champagne que dans le Canton, mais le revenu-travail du paysan arrive à égaliser ou presque celui du paysan genevois moyen: l'extension des cultures semble compenser les faibles rendements: cette terre strictement genevoise est donc plus laborieuse que d'autres communes à population plus mélangée.

Les trois composantes de la population genevoise se présentent comme suit: Genevois $(32,70 \%)$, Confédérés $(50,10 \%)$ et étrangers $(17,20 \%)$; il s'agit de moyennes pour tout le Canton, ville comprise. Or, la Champagne présente les rapports suivants: $51 \%$ de Genevois, 39\% de Confédérés et 10\% d'étrangers. C'est en Champagne que l'on repère les plus fortes concentrations relatives de Genevois, par exemple à Laconnex qui compte $66 \%$ de nationaux, Aire-la-Ville 63\%, Soral 59\%. Aire-la-Ville est la commune genevoise comptant le moins d'étrangers $(7 \%)$ et Laconnex est la commune qui a attiré le moins de Confédérés (24\%). Si l'on compare avec deux communes strictement rurales situées l'une dans le district Rhône-Lac (Satigny), on trouve: Genevois, 37,50\%, Confédérés, $41,50 \%$, étrangers, $21 \%$, alors que Choulex, située dans le district Arve-Lac, compte $50 \%$ de Genevois, $29 \%$ de Confédérés et $21 \%$ d'étrangers. Or, une loi générale veut que l'immigrant rural cherche les bonnes terres et refuse les médiocres. Le très fort pourcentage de population genevoise démontre cette règle: l'immigrant n'est jamais attiré par des terres peu fertiles. Ces communes sont peu peuplées, puisque leur densité n'est que le $37 \%$ de celle du canton rural. Les Confédérés et les étrangers ne sont pas attirés par cette région à faible densité, alors que le peuplement hypertrophique de la ville, des communes suburbaines et de quelques communes rurales pose des problèmes compliqués à résoudre.

Nous donnons un tableau très schématique des principales activités rurales du Canton de Genève, pour les comparer avec celles de la Champagne.

\begin{tabular}{|c|c|c|}
\hline & Canton & Champagne \\
\hline Surface sous blé . & $33,00 \%$ & $38,45 \%$ \\
\hline urface sous tubercule & $8,78 \%$ & $9,3+\%$ \\
\hline utres cultures & $14,70 \%$ & $6,87 \%$ \\
\hline Herbages . & $35,15 \%$ & $36,21 \%$ \\
\hline Bois et marais . & $6,80 \%$ & $6,73 \%$ \\
\hline Terrains non prod. & $2,58 \%$ & $2,40 \%$ \\
\hline
\end{tabular}

On voit que la polyculture ne semble pas être le fait du paysan de la Champagne, qui est équipé pour les travaux de la «grosse»agriculture et qui ne peut s'astreindre aux délicats soucis de la petite culture. Examinons encore quelques aspects statistiques de ce problème. 


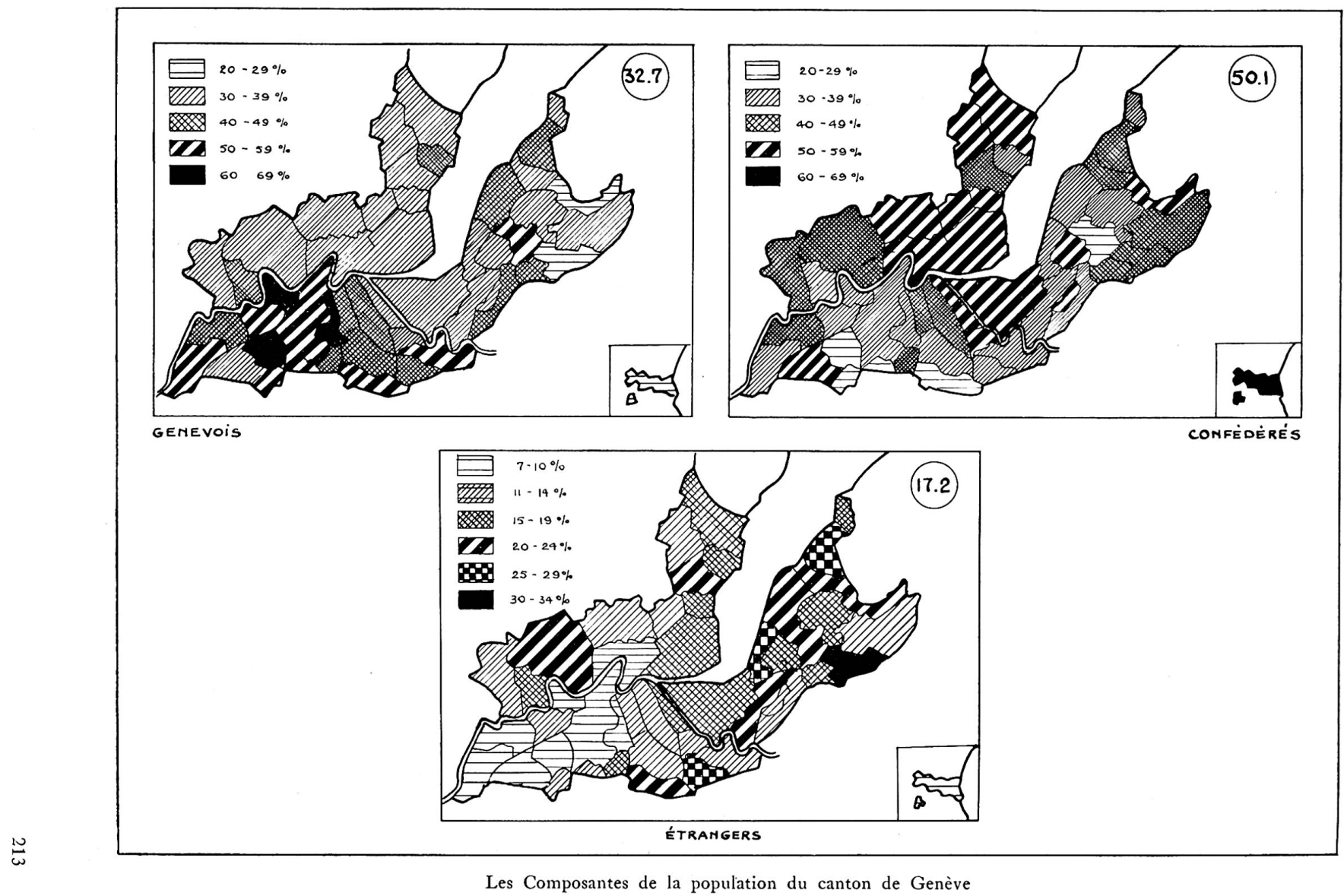




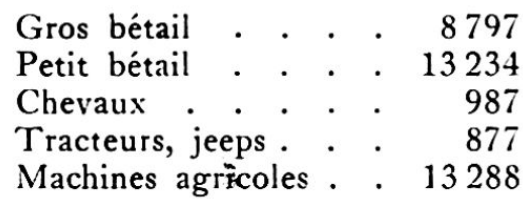

$\begin{array}{lr}6,2 & 1162 \\ 9,5 & 491 \\ 0,7 & 149 \\ r, 6 & 126 \\ 9,4 & 1769\end{array}$

13

5,6

1,7

1,4

10

Dans ce tableau consacré au cheptel mort et vif, on perçoit certaines différences notables, par exemple, dans la répartition du petit bétail, car les grandes porcheries de Plan-les-Ouates, Perly et Collex-Bossy faussent les comparaisons. Il y a plus de bétail et de chevaux en Champagne que dans le reste du Canton (proportionnellement), ainsi que les jeeps et les tracteurs; la proportion de machinerie agricole fixe et mobile est la même par exploitation, que ce soit dans le Canton ou en Champagne. Mais si l'on pousse l'analyse plus à fond, on voit que par tête de personne active on obtient les chiffres suivants:

$\begin{array}{lcc} & \text { Canton } & \text { Champagne } \\ \text { Jeeps et tracteurs } & 0,15 & 0,20 \\ \text { Machines } & 2,3 & 2,8\end{array}$

On a donc suppléé à une population active relativement faible par la machinerie fixe et mobile, le cheval jouant encore un rôle important dans cette Champagne prétéritée naturellement.

Nous avons mentionné l'éloignement du centre de la ville, compris entre 7 et 15 kilomètres. Pourquoi donc, devant l'engorgement urbain, des vagues d'émigrants, ce terme semble exact, n'iraient-elles pas s'installer dans ces terres à faible densité humaine, qui apparaissent comme des pôles d'attraction?

Mais par loi, cette région est classée comme zone agricole et ne peut être envisagée comme zone de résidence. D'autre part, les transports en commun ont été défavorablement influencés par la démographie de ces communes. Malgré les servitudes imposées par le cahier des charges à la Compagnie genevoise des tramways électriques, la Champagne a toujours été mal desservie. Si l'on analyse une carte isochronique des transports en commun, qui tient compte non seulement de la durée du trajet depuis le centre de la ville, mais encore du temps mis de la station au domicile, ce que l'on omet trop souvent dans de telles études, on constate que la Champagne est située à plus de 45 minutes de trajet de la ville. La faible population et son caractère rural, usagère irrégulière des lignes de tramways n'a guère encouragé cette compagnie, du reste victime de déficits chroniques auxquels l'Etat tente de rémédier, a beaucoup améliorer ses horaires et la fréquence des convois. Depuis 2 ans on assiste à un sérieux effort et le remplacement des vétustes trams par de pratiques autobus, la création ou le détournement de quelques lignes semblent freiner quelque peu le mouvement d'émigration vers la ville ou les communes suburbaines.

Personne ne peut songer à aller s'établir dans cette région, même déclassée en faveur d'un peuplement hypothétique, puisque toute cette zone se trouve à plus de 45 minutes de trajet de la ville. Même avec l'hypermotorisation du canton de Genève, un père de famille ne peut songer à obliger ses enfants à effectuer de tels parcours, soit pour suivre les écoles supérieures, soit pour exercer une profession. Seule la commune de Soral présente une légère augmentation depuis 1956 et encore faut-il tenir compte de l'arrivée dans cette commune d'une famille nombreuse dont le chef a pu acquérir une vaste maison à un prix intéressant. Cet exemple prouve que pour interpréter quelquefois des statistiques, il faut connaitre la chronique villageoise ....

Le Canton rural compte $48,80 \%$ d'hommes et $51,20 \%$ de femmes, alors que la Champagne compte 947 hommes et 934 femmes. La présence de quelques 40 gendarmes et garde-frontières ne joue aucun rôle dans cet aspect sexuel de la population, car la plupart sont des hommes mariés. 
Il résulte de ce qui précède que la Champagne constitue un petit monde à part, caractérisé par la porosité de son sol, sa forte population active dans l'agricultures la monoculture, les faibles rendements obtenus, l'extension des cultures, l'usage de la machinerie, la forte proportion de Genevois, et d'hommes. Devons-nous prétendre que chaque fois que le sol sera perméable et graveleux sur le $60 \%$ de la surface d'une région étudiée, ces conditions seront remplies. Il serait ridicule de le prétendre. La nature du sol a créé un état de fait qui ressort de l'analyse, mais qui n'a rien de directement influencé. Il s'agit de conséquences lointaines. Mais on peut reconnaître que ce terroir, s'il était très éloigné d'un centre important, serait abandonné ou presque désertique. La proximité d'un centre tel que Genève a été malgré tout un frein à l'émigration, tout en attirant quelques habitants. Un tel marché reste intéressant pour le paysan et l'intérêt que Genève a toujours montré pour l'agronomie et la mise sous culture de ses terres si resteintes en surface a aidé la Champagne à ne pas perdre courage.

Quels remèdes pourrait-on suggérer? L'irrigation est impossible, même avec la présence du Rhône qui limite au Nord et à l'Ouest ces sept communes. Outre que son niveau est à $85 \mathrm{~m}$. en dessous du plateau, on peut admettre que les frais de pompage et de répartition de l'eau seraient trop onéreux. On pourrait aussi, ce qui fut fait, proposer l'utilisation des nappes d'eau souterraines devinées théoriquement par Etienne Joukowsky et découvertes pratiquement par les sondages des Services industriels de Genève. Seule la nappe inférieure est exploitée par le Service des Eaux, parce qu'elle n'est pas contaminée. Mais il faut conserver les nappes phréatiques pour la consommation humaine et industrielle et il est hors de question de pomper l'eau accumulée dans le réseau hydrographique préquaternaire creusé dans la molasse et dont les vallées furent comblées par les dépôts quaternaires, graviers, argiles, avec au-dessus les dépôts superficiels décrits plus haut.

La technique moderne, la génétique, la mécanisation, la culture atomique même ne pourront jamais rendre impérméable un sol de graviers. Et au fur et à mesure que l'agronomie, cette science chère de tout temps aux Genevois, progressera encore grâce aux chaires et aux laboratoires de l'Université, la Champagne verra augmenter la différence entre ses rendements et ceux des communes à terres fortes, avec tout ce que ces déficits peuvent apporter de modifications démographiques.

Mais on ne peut désespérer. Les impérieux besoins techniques actuels nés du vaste programme de travaux publics de l'Etat de Genève, les projets routiers, l'allongement et le renforcement des pistes de l'aérodrome de Cointrin, amènent un élément de prospérité inattendu. Les tas de graviers extraits du Rhône lors de la construction de l'usine hydro-électrique de Verbois, qui déshonoraient l'aimable paysage rhodanien, disparaissent, emportés par d'habiles petits entrepreneurs valaisans, qui ouvrent aussi des gravières sur le plateau. Les sables du Cannelet s'avèrent d'une rentabilité insoupçonnée.

La physionomie de la Champagne va lentement se modifier. La structure économique va peut-être se transformer et le gravier, qui trop longtemps fut un frein au développement de ce charmant terroir aux villages chers aux Genevois, ce gravier peut devenir un élément de prospérité.

\section{DER EINFLUSS DES BODENS AUF BEVÖLKERUNG UND WIRTSCHAFT EINER GENFER REGION, DER CHAMPAGNE}

Die kleine Genfer Landschaft Champagne $\left(29 \mathrm{~km}^{2}, 15 \%\right.$ der Kantonsfläche, 1911 Bewohner, $5 \%$ der Kantonsfläche) zeichnet sich durch eine auffällig geringe Volksdichte (66 gegen 176 des ganzen ländlichen Kantonsteils) aus. Ein Hauptgrund liegt im Vorherrschen trockener weil durchlässiger Kiesböden, die $60 \%$ der Gesamtfläche der Champagne einnehmen und nur $70 \%$ der Getreideund $75 \%$ der Kartoffelerträge des Ertragsmittels der übrigen Kantonsteile liefern. Damit hängt nicht zuletzt die Entvölkerung des Gebietes (1900-1950: 25\%) zusammen, das andrerseits dafür ein $\propto$ Pol» der Abwehr fremder Elemente blieb. Industrie und Verkehr (Flugplatz) werden der Champagne jedoch erneuten Auftrieb bringen, was der liebenswerten Landschaft sehr zu wünschen ist. 